\title{
BMJ Open The association between adverse childhood experiences and traumatic brain injury/concussion in adulthood: A scoping review protocol
}

\author{
Zechen Ma, ${ }^{1}$ Mark T Bayley, ${ }^{1}$ Laure Perrier, ${ }^{2}$ Priya Dhir, ${ }^{3,4}$ Lana Dépatie, ${ }^{4}$ \\ Paul Comper, ${ }^{1}$ Lesley Ruttan, ${ }^{1}$ Sarah EP Munce ${ }^{1}$
}

To cite: Ma Z, Bayley MT, Perrier L, et al. The association between adverse childhood experiences and traumatic brain injury/concussion in adulthood: A scoping review protocol. BMJ Open 2017;7:e018425. doi:10.1136/ bmjopen-2017-018425

- Prepublication history and additional material for this paper are available online. To view these files, please visit the journal online (http://dx.doi. org/10.1136/bmjopen-2017018425).

Received 28 June 2017 Revised 8 August 2017 Accepted 18 September 2017

CrossMark

${ }^{1}$ Hull-Ellis Concussion Research Centre, Toronto Rehabilitation Institute, University Health Network, Toronto, Ontario, Canada

${ }^{2}$ Gerstein Science Information Centre, University of Toronto, Toronto, Ontario, Canada ${ }^{3}$ Faculty of Medicine, University of Toronto, Toronto, Ontario,

Canada

${ }^{4}$ The Hospital for Sick Children, Toronto, Ontario, Canada

Correspondence to Dr Sarah EP Munce; sarah.munce@uhn.ca

\section{ABSTRACT}

Introduction Exposure to adverse childhood experiences (ACEs) is a significant risk factor for physical and mental illnesses later in life. Concussion or traumatic brain injury is a challenging condition where preinjury factors may interact to affect recovery. The association between ACEs and traumatic brain injury/concussion is not well mapped in any previous reviews of the literature. Using a scoping review methodology, the research question that will be addressed is: what is known from the existing literature about the association between ACEs and traumatic brain injury/concussion in adults?

Methods and analysis The methodological frameworks outlined by Arksey and O'Malley and Levac et a/ will be used. All original studies in English published since 2007 investigating ACEs and traumatic brain injury/concussion outcomes will be included with no limitations on study type. Literature search strategies will be developed using medical subject headings and text words related to ACEs and traumatic brain injury/concussions. Multiple electronic databases will be searched. Two independent reviewers will screen titles and abstracts for full-text review and full texts for final inclusion. Two independent reviewers will extract data on study characteristics for ACE exposure and traumatic brain injury/concussion outcomes. Extracted data will be summarised quantitatively using numerical counts and qualitatively using thematic analysis.

Dissemination This review will identify knowledge gaps on the associations between ACEs and traumatic brain injury/concussion and promote further research. Knowledge translation will occur throughout the review process with dissemination of project findings to stakeholders at the local, national and international levels.

\section{INTRODUCTION}

Adverse childhood experiences (ACEs) are typically defined as stress or trauma occurring in the first 18 years of life. Felitti and colleagues ${ }^{1}$ first described ACEs and defined it as exposure to psychological, physical or sexual abuse, and household dysfunction including substance abuse (problem drinking/alcoholic and/or street drugs), mental illness, a mother treated violently and
Strengths and limitations of this study:

- No previous research has systematically mapped the association between adverse childhood experiences and traumatic brain injury/concussion.

- This review will be conducted using an exhaustive search strategy guided by an experienced information specialist.

- The review will include studies of all study designs to capture the full range of evidence.

- This review will be limited to English language studies only.

- The search may yield self-reported data, which are subject to response bias.

criminal behaviour in the household. ${ }^{1}$ Along with the initial ACE study, other studies have characterised ACEs as neglect, parental separation, loss of family members or friends, long-term financial adversity and witness to violence. ${ }^{23}$ From the original cohort of 9508 American adults, more than half of respondents $(52 \%)$ experienced at least one adverse childhood event. ${ }^{1}$ Since the original cohort, ACE exposures have been investigated globally revealing comparable prevalence to the original cohort. ${ }^{45}$ More recently in 2014, a survey of 4000 American children found that $60.8 \%$ of children had at least one form of direct experience of violence, crime or abuse. ${ }^{6}$ The ACE study precipitated interest in the health conditions of adults maltreated as children as it revealed links to chronic diseases such as obesity, autoimmune diseases, heart, lung and liver diseases, and cancer in adulthood. ${ }^{1}$ Since then, further evidence has revealed relationships between ACEs and physical and mental health outcomes, such as increased risk of substance abuse, suicide and premature mortality. ${ }^{47}$

Individual studies have also shown that ACEs are positively associated with self-reported 
disability, chronic pain, depression and headaches. ${ }^{8-12}$ Numerous structural MRI studies on maltreated children compared with control groups have shown that childhood abuse promotes long-term changes in brain development, and specifically structural impairments in areas involved in cognitive and emotional functioning such as the prefrontal cortex and limbic system. ${ }^{13-17}$ In addition, functional studies support the structural findings as maltreated children experience deficits in executive functioning and demonstrate hypervigilant responses to emotional threats, shown through variations in neural activation. ${ }^{17-19}$

Concussion and traumatic brain injury are challenging and controversial conditions where previous research has identified that preinjury pain and psychological issues, such as anxiety and mood disorders, may affect recovery. ${ }^{20-22}$ Furthermore, a cumulative stressor model has been proposed to explain the aetiology of persistent postconcussion symptoms where stressors interact with premorbid health resulting in prolonged symptoms. ${ }^{23}$ While previous systematic reviews have examined the associations between ACEs and various conditions including obesity ${ }^{24}$ cardiovascular disease risk, ${ }^{25}$ type 2 diabetes, ${ }^{26}$ cancer, ${ }^{27}$ asthma, ${ }^{28}$ schizotypy, ${ }^{29}$ psychotic symptoms ${ }^{30}$ and adult sleep disorders, ${ }^{31}$ to date, no reviews have been conducted on the associations between ACEs and traumatic brain injury/concussion. Thus, the specific research question of this scoping review is the following: what is known from the existing literature about the association between ACEs and traumatic brain injury/concussion in adults?

\section{METHODS AND ANALYSIS}

We will use the methodological frameworks proposed by Arksey and O'Malley as well as Levac and colleagues for the current scoping review. ${ }^{32} 33$ These frameworks outline six different stages involved in a scoping review: (1) identifying the research question; (2) identifying relevant studies; (3) selecting studies; (4) charting the data; (5) collating, summarising and reporting the results; and (6) consulting with relevant stakeholders. ${ }^{32}{ }^{33}$ The research team has experience/expertise in ACEs (LD, PD), traumatic brain injury/concussion (SEPM, MTB, LR) and knowledge synthesis methods (SEPM, LP, ZM, MTB). Although traditionally applied to systematic review protocols, the preferred reporting items for systematic reviews and meta-analysis for protocols (PRIMSA-P) was used to draft this protocol. ${ }^{34}$

\section{Eligibility criteria}

For the purpose of this review, we will be using the definition of ACEs as outlined in the original ACEs study by Felitti and colleagues as it guided successive primary research and reviews on childhood adversity. ${ }^{15} 71027$ ACEs are defined as stressful or traumatic life events that occur during the first 18 years of life, such as psychological, physical or sexual abuse. ${ }^{1}$ ACEs may also include exposure to various aspects of household dysfunction such as substance abuse (problem drinking/alcoholic and/or street drugs), mental illness, a mother treated violently and criminal behaviour in the household. ${ }^{1}$ The specific behaviours are detailed in Felitti et als ACEs questionnaire. ${ }^{1}$ ACEs may be assessed using self-report, family member reporting or information extracted from police records, which is consistent with a previous systematic review on ACEs and adult sleep disorders. ${ }^{31}$ The outcome of interest will be any measure of traumatic brain injury or concussion defined as any injury to the head arising from blunt or penetrating trauma that manifests in changes in consciousness, memory or anatomical features. ${ }^{35}$ Studies will be included where at least $50 \%$ of the sample includes individuals who are $\geq 18$ years old of age at the time of traumatic brain injury or concussion. This outcome may be assessed subjectively, objectively or via clinical diagnosis. Furthermore, primary research published in the last 10 years (2007 to search date) of all study designs will be considered for inclusion (eg, observational studies, randomised controlled trials and qualitative studies). Systematic reviews, meta-analyses, editorials, commentaries and conference proceedings will not be included. Furthermore, only studies reported in English will be included.

\section{Search strategy and information sources}

Literature search strategies will be developed using medical subject headings (MeSH) and text words related to ACEs and concussion/traumatic brain injury. The search strategy will be developed by an information specialist (LP) with expertise in systematic and scoping reviews. The search will be conducted in MEDLINE (OVID interface), CINAHL (EBSCO interface), EMBASE (OVID interface), PsycINFO (OVID interface) and the Cochrane Central Register of Controlled Trials (Cochrane Library). The search strategy for MEDLINE can be found in the (online supplementary additional file). To ensure literature saturation, a hand search of the reference lists from reviews and selected articles will be completed. Finally, experts in the field of ACEs will be contacted and consulted in order to ensure that all relevant data are obtained, including members of the research team (LD, MB).

\section{Data management}

Literature search results will be uploaded to Covidence, a web-based software that facilitates collaboration among reviewers during the screening and data abstraction phases. The team will develop inclusion and exclusion criteria screening questions and forms for title and abstract screening (level 1 screening) and full-text screening (level 2 screening).

\section{Study selection}

Two reviewers (ZM \& PD) will independently screen the titles and abstracts identified by the literature search for inclusion using the screening form. To promote the 
inter-rater reliability, a pilot test of the level 1 screening form based on the aforementioned criteria will be conducted on a random sample of approximately 100 articles. The $\kappa$ statistic will then be calculated to determine the inter-rater agreement. ${ }^{36}$ If low agreement is observed, the inclusion and exclusion criteria will be modified to improve the consistent application of the selection criteria. After level 1 screening, the full text of potentially relevant articles will be collected and two independent reviewers will screen to determine final inclusion. A pilot test of the level 2 screening form will be performed on approximately $1 \%$ of the articles and the inter-rater reliability for study inclusion will also be calculated. ${ }^{36}$ Discrepancies will be resolved by discussions, and if necessary, by a third reviewer who is knowledgeable in the research area. Studies excluded during the screening phases will be documented in Covidence along with an explanation for the exclusion.

\section{Data items and data collection process}

Abstracted data will include the following characteristics: author, year of publication, sample size, mean age, exposure/type of ACE (eg, psychological, physical and sexual abuse), traumatic brain injury/concussion-related outcome (ie, measure), covariates and main findings. Additional categories may be identified through the completion of the search and through discussions with the research team and key stakeholders. To ensure consistency across reviewers, the data abstraction form will also be pilot tested and modified if poor agreement is observed. For example, any wording on the form that may be related to poor agreement will be reviewed and clarified. Two reviewers will independently abstract all of the data from eligible studies. Disagreements will be resolved through discussion or third reviewer adjudication. The quality of evidence will not be evaluated as the purpose of a scoping review is to identify gaps in the literature and future areas for primary research or for a systematic review. 3334

\section{Synthesis}

The data from this scoping review will be summarised quantitatively using numerical counts and qualitatively using thematic analysis. When appropriate, data will be grouped by type of ACE. This scoping review will identify research gaps as well as opportunities for future study either via implementation studies, consensus meeting or systematic review. Furthermore, information gleaned from this review will contribute to research and intervention development that address the needs of survivors.

\section{DISCUSSION AND DISSEMINATION}

As scoping reviews do not assess the quality of evidence and risk of bias of included studies, the findings from this review will be subject to the strengths and limitations of the included studies. ${ }^{33} 34$ The research question seeks out a temporal relationship between ACEs and subsequent traumatic brain injury in adulthood that may not be well captured in the existing literature. For example, this requires authors to distinguish between traumatic brain injury incurred from childhood physical abuse and adulthood traumatic brain injury. Additionally, the search may yield studies based on self-reported and retrospective recall data that are widely used in epidemiological studies investigating ACEs. ${ }^{135-9} 37$

Knowledge translation activities will occur from the initial stages of the review and continue throughout with dissemination of the research question to key stakeholders such as the Ontario Neurotrauma Foundation and March of Dimes Canada. Consultation with these organisations and other similar organisations is consistent with the final stage of the scoping review frameworks. ${ }^{33}$ These activities will help to ensure that our results and their interpretations are discussed among other experts in the field and disseminated to the appropriate audiences. End-of-grant knowledge translation will also take place through these stakeholders, their outlets (eg, print and online newsletters), as well as through conventional knowledge translation mechanisms (eg, peer-reviewed journals and conference). For example, the results of the scoping review could be presented at meetings locally, nationally and internationally (eg, International Brain Injury Association Conference in 2019). Additionally, the scoping review will be published in a peer-reviewed journal to present results to the appropriate academic and clinical audiences. Finally, the results of this scoping review will inform the standardised data collection strategies in collaboration with local clinical programme and/or research initiatives (eg, the Hull-Ellis Concussion and Research Clinic at Toronto Rehabilitation Institute, University Health Network).

Contributors SEPM: conceived of the scoping review, together with MTB, LP, ZM, and PD. SEPM and ZM: wrote the first draft of the protocol and are the guarantors of the review. SEPM, LP, ZM and LD: involved in the preliminary literature review. LP: conducted the literature search and provided methodological expertise (knowledge synthesis). LD, PC, LR and MTB: provided critical content expertise on adverse childhood experiences/traumatic brain injury/concussion that was integrated into the current protocol. All authors: involved in editing and revising the protocol for important intellectual content and approved the final version of the protocol.

Funding SEPM has been supported by a Heart and Stroke Foundation of Canada Focus on Stroke Fellowship, Toronto Rehabilitation Institute, University Health Network, Canadian Institutes of Health Research Fellowship, and a European Stroke Research Foundation Investigator Award. ZM has been supported by the Institute of Medical Sciences at the University of Toronto under the Summer Undergraduate Research Program and by the University Health Network.

Competing interests None declared.

Provenance and peer review Not commissioned; externally peer reviewed.

Open Access This is an Open Access article distributed in accordance with the Creative Commons Attribution Non Commercial (CC BY-NC 4.0) license, which permits others to distribute, remix, adapt, build upon this work non-commercially, and license their derivative works on different terms, provided the original work is properly cited and the use is non-commercial. See: http://creativecommons.org/ licenses/by-nc/4.0/

(c) Article author(s) (or their employer(s) unless otherwise stated in the text of the article) 2017. All rights reserved. No commercial use is permitted unless otherwise expressly granted. 


\section{REFERENCES}

1. Felitti VJ, Anda RF, Nordenberg D, et al. Relationship of childhood abuse and household dysfunction to many of the leading causes of death in adults. The Adverse Childhood Experiences (ACE) Study. Am J Prev Med 1998;14:245-58.

2. Bernstein DP, Fink L, Handelsman L, et al. Initial reliability and validity of a new retrospective measure of child abuse and neglect. $A m \mathrm{~J}$ Psychiatry 1994;151:1132-6.

3. Finkelhor D, Hamby SL, Ormrod R, et al. The juvenile victimization questionnaire: reliability, validity, and national norms. Child Abuse Negl 2005;29:383-412.

4. Bellis MA, Lowey $\mathrm{H}$, Leckenby $\mathrm{N}$, et al. Adverse childhood experiences: retrospective study to determine their impact on adult health behaviours and health outcomes in a UK population. J Public Health 2014;36:81-91.

5. Soares AL, Howe LD, Matijasevich A, et al. Adverse childhood experiences: Prevalence and related factors in adolescents of a Brazilian birth cohort. Child Abuse Negl 2016;51:21-30.

6. Finkelhor D, Turner HA, Shattuck A, et al. Prevalence of Childhood Exposure to Violence, Crime, and Abuse: Results From the National Survey of Children's Exposure to Violence. JAMA Pediatr 2015;169:746-54.

7. Brown DW, Anda RF, Tiemeier H, et al. Adverse childhood experiences and the risk of premature mortality. Am J Prev Med 2009;37:389-96.

8. Schüssler-Fiorenza Rose SM, Eslinger JG, Zimmerman L, et al. Adverse Childhood Experiences, Support, and the Perception of Ability to Work in Adults with Disability. PLoS One 2016;11:e0157726.

9. Sachs-Ericsson N, Kendall-Tackett K, Hernandez A. Childhood abuse, chronic pain, and depression in the National Comorbidity Survey. Child Abuse Negl 2007;31:531-47.

10. Chapman DP, Whitfield CL, Felitti VJ, et al. Adverse childhood experiences and the risk of depressive disorders in adulthood. $J$ Affect Disord 2004;82:217-25.

11. Anda RF, Whitfield CL, Felitti VJ, et al. Adverse childhood experiences, alcoholic parents, and later risk of alcoholism and depression. Psychiatr Serv 2002;53:1001-9.

12. Anda R, Tietjen G, Schulman E, et al. Adverse childhood experiences and frequent headaches in adults. Headache 2010;50:1473-81.

13. Hanson JL, Nacewicz BM, Sutterer MJ, et al. Behavioral problems after early life stress: contributions of the hippocampus and amygdala. Biol Psychiatry 2015;77:314-23.

14. Whittle S, Dennison M, Vijayakumar N, et al. Childhood maltreatment and psychopathology affect brain development during adolescence. J Am Acad Child Adolesc Psychiatry 2013;52:940-52.

15. De Bellis MD, Keshavan MS, Shifflett $\mathrm{H}$, et al. Brain structures in pediatric maltreatment-related posttraumatic stress disorder: a sociodemographically matched study. Biol Psychiatry 2002;52:1066-78.

16. Carrion VG, Weems CF, Watson C, et al. Converging evidence for abnormalities of the prefrontal cortex and evaluation of midsagitta structures in pediatric posttraumatic stress disorder: an MRI study. Psychiatry Res 2009;172:226-34.

17. De Brito SA, Viding E, Sebastian CL, et al. Reduced orbitofrontal and temporal grey matter in a community sample of maltreated children. J Child Psychol Psychiatry 2013;54:105-12.
18. De Bellis MD, Hooper SR. Neural substrates for processing task-irrelevant emotional distracters in maltreated adolescents with depressive disorders: a pilot study. $J$ Trauma Stress 2012;25:198-202.

19. Bruce J, Fisher PA, Graham AM, et al. Patterns of brain activation in foster children and nonmaltreated children during an inhibitory control task. Dev Psychopathol 2013;25:931-41.

20. Silverberg ND, Iverson GL. Etiology of the post-concussion syndrome: Physiogenesis and Psychogenesis revisited. NeuroRehabilitation 2011;29:317-29.

21. Ponsford J, Cameron P, Fitzgerald M, et al. Predictors of postconcussive symptoms 3 months after mild traumatic brain injury. Neuropsychology 2012;26:304-13.

22. Evered L, Ruff R, Baldo J, et al. Emotional risk factors and postconcussional disorder. Assessment 2003;10:420-7.

23. Iverson GL. Outcome from mild traumatic brain injury. Curr Opin Psychiatry 2005;18:301-17.

24. Danese A, Tan M. Childhood maltreatment and obesity: systematic review and meta-analysis. Mol Psychiatry 2014;19:544-54.

25. Su S, Jimenez MP, Roberts CT, et al. The role of adverse childhood experiences in cardiovascular disease risk: a review with emphasis on plausible mechanisms. Curr Cardiol Rep 2015;17:88.

26. Huang $\mathrm{H}$, Yan $\mathrm{P}$, Shan $\mathrm{Z}$, et al. Adverse childhood experiences and risk of type 2 diabetes: A systematic review and meta-analysis. Metabolism 2015;64:1408-18.

27. Holman DM, Ports KA, Buchanan ND, et al. The Association Between Adverse Childhood Experiences and Risk of Cancer in Adulthood: A Systematic Review of the Literature. Pediatrics 2016;138(Suppl 1):S81-91.

28. Exley D, Norman A, Hyland M. Adverse childhood experience and asthma onset: a systematic review. Eur Respir Rev 2015;24:299-305.

29. Velikonja T, Fisher HL, Mason O, et al. Childhood trauma and schizotypy: a systematic literature review. Psychol Med 2015;45:947-63.

30. Trotta A, Murray RM, Fisher HL. The impact of childhood adversity on the persistence of psychotic symptoms: a systematic review and meta-analysis. Psychol Med 2015;45:2481-98.

31. Kajeepeta S, Gelaye B, Jackson CL, et al. Adverse childhood experiences are associated with adult sleep disorders: a systematic review. Sleep Med 2015;16:320-30.

32. Arksey H, O'Malley L. Scoping studies: towards a methodological framework. Int J Soc Res Methodol 2005;8:19-32.

33. Levac D, Colquhoun H, O'Brien KK. Scoping studies: advancing the methodology. Implement Sci 2010;5:69.

34. Shamseer L, Moher D, Clarke M, et al. Preferred reporting items for systematic review and meta-analysis protocols (PRISMA-P) 2015: elaboration and explanation. BMJ 2015;349:g7647.

35. Menon DK, Schwab K, Wright DW, et al. Position statement: definition of traumatic brain injury. Arch Phys Med Rehabil 2010;91:1637-40.

36. Landis JR, Koch GG. The measurement of observer agreement for categorical data. Biometrics 1977;33:159-74.

37. Hardt J, Rutter M. Validity of adult retrospective reports of adverse childhood experiences: review of the evidence. J Child Psychol Psychiatry 2004;45:260-73. 